\title{
Periodic Solutions of Multispecies Mutualism System with Infinite Delays
}

\author{
Wenbo Zhao, ${ }^{1}$ Caocuan $\mathrm{Ma},{ }^{2}$ Taotao Zheng, ${ }^{3}$ and Xiao-Ke Sun ${ }^{2}$ \\ ${ }^{1}$ School of Physics and Information, Tianshui Normal University, Tianshui, Gansu 741000, China \\ ${ }^{2}$ School of Mathematics and Statistics, Tianshui Normal University, Tianshui, Gansu 741000, China \\ ${ }^{3}$ Department of Mathematics, Zhejiang University, Hangzhou, Zhejiang 310000, China
}

Correspondence should be addressed to Wenbo Zhao; zhao_wenbo@yahoo.com

Received 27 June 2014; Accepted 9 August 2014; Published 24 August 2014

Academic Editor: Yonghui Xia

Copyright (C) 2014 Wenbo Zhao et al. This is an open access article distributed under the Creative Commons Attribution License, which permits unrestricted use, distribution, and reproduction in any medium, provided the original work is properly cited.

\begin{abstract}
We studied the delayed periodic mutualism system with Gilpin-Ayala effect. Some new and interesting sufficient conditions are obtained to guarantee the existence of periodic solution for the multispecies mutualism system with infinite delays. Our method is based on Mawhin's coincidence degree. To the best knowledge of the authors, there is no paper considering the existence of periodic solutions for $n$-species mutualism system with infinite delays.
\end{abstract}

\section{Introduction}

Recently, there are many papers considering the existence of periodic solutions for competitive Lotka-Volterra system based on Mawhin's coincidence degree theory (see [1-4]). But there are few papers considering the periodicity of mutualism system; for example, one can refer to [5-7]. However, the references mentioned above only considered two-dimensional mutualism system. To the best knowledge of the authors, there is no paper considering the existence of periodic solutions for $n$-species mutualism system. It should be noted that the method used in [5-7] is difficult to be extended to the $n$-dimensional system. So, we employ the method used in [2-4]. However, the problem considered in this paper is completely different from those mentioned above. On the other hand, the above-mentioned works considered the models with constant discrete delays or without delays. In practice, there will be a distribution of transmission delays. In this case, the transmission of species is no longer instantaneous and cannot be modelled with discrete delays. A more appropriate way is to incorporate distributed delays. Therefore, the studies of the model with distributed delays have more important significance than the ones of the model with discrete delays. Thus, in this paper, we considered the following mutualism system with distributed delays:

$$
\begin{array}{r}
\dot{y}_{i}(t)=y_{i}(t)\left[r_{i}(t)-a_{i i}(t) y_{i}(t)^{\alpha_{i j}}(t)+\sum_{j=1, j \neq i}^{n} a_{i j}(t) y_{j}^{\alpha_{i j}}(t)\right. \\
+\sum_{j=1, j \neq i}^{n} b_{i j}(t) y_{j}^{\beta_{i j}}\left(t-\tau_{i j}\right) \\
\left.+\sum_{j=1, j \neq i}^{n} c_{i j}(t) \int_{0}^{\infty} H_{i j}(s) y_{j}^{\gamma_{i j}}(t+s) d s\right], \\
i=1,2, \ldots n,
\end{array}
$$

where $r_{i}, a_{i j}, b_{i j}$, and $c_{i j}, i, j=1,2, \ldots, n$, are $\omega$-periodic functions, that is, $r_{i}(t+\omega)=r_{i}(t), a_{i j}(t+\omega)=a_{i j}(t)$, $b_{i j}(t+\omega)=b_{i j}(t)$, and $c_{i j}(t+\omega)=c_{i j}(t)$, and $\alpha_{i j}, \beta_{i j}$, and $\gamma_{i j}$ are constants, $i, j=1,2, \ldots, n$. From biological view, $r_{i}, a_{i j}, b_{i j}$, and $c_{i j}, i, j=1,2, \ldots, n$, are nonnegative, $\int_{0}^{\infty} H_{i j}(s) d s=1$, 
and $a_{i i}$ is positive. System (1) is associated with the IVP as follows:

$$
y_{i}\left(t_{0}\right)=y_{i}^{0}, \quad y_{i}^{0}>0, i=1,2, \ldots n .
$$

\section{Existence of Periodic Solutions}

For convenience, we introduce some notations, definitions, and lemmas. If $g(t)$ is a continuous $\omega$-periodic function defined on $\mathbf{R}$, denote

$$
\begin{gathered}
\underline{g}=\min _{t \in[0, \omega]}|g(t)|, \quad \bar{g}=\max _{t \in[0, \omega]}|g(t)|, \\
m(g)=\frac{1}{\omega} \int_{0}^{\omega} g(t) d t .
\end{gathered}
$$

We also denote the spectral radius of the matrix $\mathscr{A}$ by $\rho(\mathscr{A})$. Denote

$$
\begin{aligned}
& X=\left\{x(t)=\left(x_{1}(t), x_{2}(t), \ldots, x_{n}(t)\right)^{T}\right. \\
&\left.\in C^{1}\left(\mathbf{R}, \mathbf{R}^{n}\right) \mid x(t+\omega)=x(t) \forall t \in \mathbf{R}\right\}, \\
& Z=\left\{x(t)=\left(x_{1}(t), x_{2}(t), \ldots, x_{n}(t)\right)^{T}\right. \\
&\left.\in C\left(\mathbf{R}, \mathbf{R}^{n}\right) \mid x(t+\omega)=x(t) \forall t \in \mathbf{R}\right\} .
\end{aligned}
$$

Lemma 1 (see [8]). Let $\Omega \subset X$ be an open and bounded set. Let $L$ be a Fredholm mapping of index zero and $N$ be L-compact on $\bar{\Omega}$ (i.e., $Q N(\bar{\Omega})$ is bounded and $K_{P}(I-Q) N: \bar{\Omega} \rightarrow X$ is compact). Assume

(i) for each $\lambda \in(0,1), x \in \partial \Omega \cap \operatorname{Dom} L, L x \neq \lambda N x$;

(ii) for each $x \in \partial \Omega \cap \operatorname{Ker} L, Q N x \neq 0$ and $\operatorname{deg}\{J Q N, \Omega \cap$ Ker $L, 0\} \neq 0$.

Then $L x=N x$ has at least one solution in $\bar{\Omega} \cap \operatorname{Dom} L$.

Definition 2 (see $[9,10])$. A real $n \times n$ matrix $\mathscr{A}=\left(a_{i j}\right)$ is said to be an $M$-matrix if $a_{i j} \leq 0, i, j=1,2, \ldots, n, i \neq j$, and $\mathscr{A}^{-1} \geq 0$.

Lemma 3 (see $[9,10])$. Let $\mathscr{A} \geq 0$ be an $n \times n$ matrix and $\rho(\mathscr{A})<1$; then $\left(E_{n}-\mathscr{A}\right)^{-1} \geq 0$, where $E_{n}$ denotes the identity matrix of size $n$.

Theorem 4. Assume the following.

$\left(H_{1}\right)$ The algebraic equation

$$
\begin{aligned}
f(u):=\left(m\left(r_{i}\right)-m\left(a_{i i}\right) u_{i}^{\alpha_{i i}}+\sum_{j=1}^{n} m\left(a_{i j}\right) u_{j}^{\alpha_{i j}}\right. \\
\left.\quad+\sum_{j=1}^{n} m\left(b_{i j}\right) u_{j}^{\beta_{i j}}+\sum_{j=1, j \neq i}^{n} m\left(c_{i j}\right) u_{j}^{\gamma_{i j}}\right)_{n \times 1}=0
\end{aligned}
$$

has finite solutions $\left(u_{1}^{*}, u_{2}^{*}, \ldots, u_{n}^{*}\right)^{T} \in \mathbf{R}_{+}^{n}$ with $u_{i}^{*}>0$ and $\sum_{u^{*}} \operatorname{sgn} J_{f}\left(u^{*}\right) \neq 0$.

$$
\begin{aligned}
& \left(H_{2}\right) \alpha_{j i} \leq \alpha_{i i}, \beta_{j i} \leq \alpha_{i i}, \gamma_{j i} \leq \gamma_{i i}(j \neq i), i, j=1,2, \ldots, n . \\
& \left(H_{3}\right) \rho(\mathscr{K})<1 \text {, where } \mathscr{K}=\left(\Gamma_{i j}\right)_{n \times n} \text { and }
\end{aligned}
$$

$$
\Gamma_{i j}= \begin{cases}0, & i=j, \\ \frac{\bar{a}_{i j}+\bar{b}_{i j}+\bar{c}_{i j}}{\underline{a}_{j j}}, & i \neq j .\end{cases}
$$

Then system (1) has at least one positive $\omega$-periodic solution.

Proof. Note that every solution $y(t)=\left(y_{1}(t), y_{2}(t), \ldots y_{n}(t)\right)^{T}$ of system (1) with the initial value condition is positive. Make the change of variables

$$
y_{i}(t)=e^{x_{i}(t)}, \quad i=1,2, \ldots, n .
$$

Then system (1) is the same as

$$
\begin{aligned}
& x_{i}(t)= r_{i}(t)-a_{i i}(t) e^{\alpha_{i j}}(t)+\sum_{j=1, j \neq i}^{n} a_{i j}(t) e^{\alpha_{i j}}(t) \\
&+\sum_{j=1, j \neq i}^{n} b_{i j}(t) e^{\beta_{i j}}\left(t-\tau_{i j}\right) \\
&+\sum_{j=1, j \neq i}^{n} c_{i j}(t) \int_{0}^{\infty} H_{i j}(s) e^{\gamma_{i j}}(t+s) d s, \\
& i=1,2, \ldots n .
\end{aligned}
$$

Obviously, if system (8) has at least one $\omega$-periodic solution, then system (1) has at least one $\omega$-periodic solution. To prove Theorem 4, we should find an appropriate open set $\Omega$ satisfying Lemma 1 . We divide the proof into three steps.

Step 1. We verify that (i) of Lemma 1 is satisfied. For any $x(t) \epsilon$ $X$, by periodicity, it is easy to check that

$$
\begin{aligned}
\Delta_{i}(x, t)= & r_{i}(t)-a_{i i}(t) e^{\alpha_{i j}}(t)+\sum_{j=1, j \neq i}^{n} a_{i j}(t) e^{\alpha_{i j}}(t) \\
& +\sum_{j=1, j \neq i}^{n} b_{i j}(t) e^{\beta_{i j}}\left(t-\tau_{i j}\right) \\
& +\sum_{j=1, j \neq i}^{n} c_{i j}(t) \int_{0}^{\infty} H_{i j}(s) e^{\gamma_{i j}}(t+s) d s .
\end{aligned}
$$

And define $L: \operatorname{Dom} L \subset X \rightarrow Z$ and $N: X \rightarrow Z$ as follows:

$$
\begin{aligned}
& X \ni x(t) \longrightarrow(L x)(t)=\frac{d x(t)}{d t} \in Z, \\
& X \ni x(t) \longrightarrow \\
& \quad(N x)(t)=\left((N x)_{1}(t),(N x)_{2}(t), \ldots,(N x)_{n}(t)\right)^{T} \in Z,
\end{aligned}
$$

where

$$
(N x)_{i}(t)=\Delta_{i}(x, t), \quad i=1,2, \ldots, n .
$$


The projectors are defined by $P: X \rightarrow X$ and $Q: Z \rightarrow Z$ by

$$
\begin{aligned}
P x(t)=\frac{1}{\omega} \int_{0}^{\omega} x(t) d t, \quad Q z(t) & =\frac{1}{\omega} \int_{0}^{\omega} z(t) d t, \\
x & \in X, \quad z \in Z .
\end{aligned}
$$

It is easy to follow that $L$ is a Fredholm mapping of index zero. Furthermore, the generalized inverse (to $L$ ) $K_{P}: \operatorname{Im} L \rightarrow$ Dom $L \cap \operatorname{Ker} P$ exists, which is given by

$$
K_{P}(y)=\int_{0}^{t} y(s) d s-\frac{1}{\omega} \int_{0}^{\omega} \int_{0}^{t} y(s) d s d t .
$$

Then $Q N: X \rightarrow Z$ and $K_{P}(I-Q) N: X \rightarrow X$ are defined by

$$
\begin{gathered}
Q N x=\left(\frac{1}{\omega} \int_{0}^{\omega} \Delta_{1}(x, t) d t, \frac{1}{\omega} \int_{0}^{\omega} \Delta_{2}(x, t) d t,\right. \\
\left.\ldots, \frac{1}{\omega} \int_{0}^{\omega} \Delta_{n}(x, t) d t\right)^{T}, \\
K_{P}(I-Q) N x=\left(\Psi_{1}(x, t), \Psi_{2}(x, t), \ldots, \Psi_{n}(x, t)\right)^{T},
\end{gathered}
$$

where

$$
\begin{aligned}
\Psi_{k}(x, t)= & \int_{0}^{t} \Delta_{k}(x, s) d s-\frac{1}{\omega} \int_{0}^{\omega} \int_{0}^{t} \Delta_{k}(x, s) d s d t \\
& -\left(\frac{t}{\omega}-\frac{1}{2}\right) \int_{0}^{\omega} \Delta_{k}(x, s) d s, \quad k=1,2, \ldots, n .
\end{aligned}
$$

Using similar arguments to Step 1 in [2], it is easy to show that $\left(K_{P}(I-Q) N x\right)(\bar{\Omega})$ is relatively compact in the space $\left(X,\|\cdot\|_{1}\right)$.

Step 2. In this step, we are in a position to search for an appropriate open bounded subset $\Omega$ satisfying condition (i) of Lemma 1. Specifically, our aim is to search for an appropriate $h_{i}$ defined by $\Omega$ in Step 1 such that $\Omega$ satisfies condition (i) of Lemma 1. To this end, assume that $x(t) \in X$ is a solution of the equation $L x=\lambda N x$ for each $\lambda \in(0,1)$; that is,

$$
\begin{aligned}
\dot{x}_{i}(t)=\lambda\left[r_{i}(t)-a_{i i}(t) e^{\alpha_{i j}}(t)+\sum_{j=1, j \neq i}^{n} a_{i j}(t) e^{\alpha_{i j}}(t)\right. \\
+\sum_{j=1, j \neq i}^{n} b_{i j}(t) e^{\beta_{i j}}\left(t-\tau_{i j}\right) \\
\left.+\sum_{j=1, j \neq i}^{n} c_{i j}(t) \int_{0}^{\infty} H_{i j}(s) e^{\gamma_{i j}}(t+s) d s\right], \\
i=1,2, \ldots n .
\end{aligned}
$$

Since $x(t) \in X$, each $x_{i}(t), i=1,2, \ldots, n$, as components of $x(t)$, is continuously differentiable and $\omega$-periodic. In view of continuity and periodicity, there exists $t_{i} \in[0, \omega]$ such that $x_{i}\left(t_{i}\right)=\max _{t \in[0, \omega]}\left|x_{i}(t)\right|, i=1,2, \ldots, n$. Accordingly, $\dot{x}_{i}\left(t_{i}\right)=$ 0 and we arrive at

$$
\begin{array}{r}
r_{i}\left(t_{i}\right)-a_{i i}\left(t_{i}\right) e^{\alpha_{i i} x_{i}\left(t_{i}\right)}+\sum_{j=1, j \neq i}^{n} a_{i j}\left(t_{i}\right) e^{\alpha_{i j} x_{j}\left(t_{i}\right)} \\
+\sum_{j=1, j \neq i}^{n} b_{i j}\left(t_{i}\right) e^{\beta_{i j} x_{j}\left(t_{i}-\tau_{i j}\right)} \\
+\sum_{j=1, j \neq i}^{n} c_{i j}(t) \int_{0}^{\infty} H_{i j}(s) e^{\gamma_{i j} x_{j}\left(t_{i}+s\right)} d s=0, \\
i=1,2, \ldots, n .
\end{array}
$$

That is,

$$
\begin{aligned}
& a_{i i}\left(t_{i}\right) e^{\alpha_{i i} x_{i}\left(t_{i}\right)} \\
& =r_{i}\left(t_{i}\right)+\sum_{j=1, j \neq i}^{n} a_{i j}\left(t_{i}\right) e^{x_{j}\left(t_{i}\right)}+\sum_{j=1, j \neq i}^{n} b_{i j}\left(t_{i}\right) e^{\beta_{i j} x_{j}\left(t_{i}-\tau_{i j}\right)} \\
& \quad+\sum_{j=1, j \neq i}^{n} c_{i j}(t) \int_{0}^{\infty} H_{i j}(s) e^{\gamma_{i j} x_{j}\left(t_{i}+s\right)} d s, \quad i=1,2, \ldots n .
\end{aligned}
$$

Noticing that $x_{j}\left(t_{j}\right)=\max _{t \in[0, \omega]}\left|x_{j}(t)\right|$ implies

$$
x_{j}\left(t_{i}-\tau_{i j}\right) \leq x_{j}\left(t_{j}\right)
$$

It follows from $\left(\mathrm{H}_{2}\right)$ that

$$
\begin{aligned}
& \underline{a}_{i i} e^{\alpha_{i i} x_{i}\left(t_{i}\right)} \\
& \leq\left|a_{i i}\left(t_{i}\right) e^{\alpha_{i i} x_{i}\left(t_{i}\right)}\right| \\
& =\mid r_{i}\left(t_{i}\right)+\sum_{j=1, j \neq i}^{n} a_{i j}\left(t_{i}\right) e^{\alpha_{i j} x_{j}\left(t_{i}\right)}+\sum_{j=1, j \neq i}^{n} b_{i j}\left(t_{i}\right) e^{\beta_{i j} x_{j}\left(t_{i}-\tau_{i j}\right)} \\
& \quad+\sum_{j=1, j \neq i}^{n} c_{i j}(t) \int_{0}^{\infty} H_{i j}(s) e^{\gamma_{i j} x_{j}\left(t_{i}+s\right)} d s \mid \\
& \leq \bar{r}_{i}+\sum_{j=1, j \neq i}^{n} \bar{a}_{i j} e^{\alpha_{i j} x_{j}\left(t_{i}\right)}+\sum_{j=1, j \neq i}^{n} \bar{b}_{i j} e^{\beta_{i j} x_{j}\left(t_{i}-\tau_{i j}\right)} \\
& \quad+\sum_{j=1, j \neq i}^{n} \bar{c}_{i j}(t) e^{\gamma_{i j} x_{j}\left(t_{i}+s\right)} \int_{0}^{\infty} H_{i j}(s) d s \\
& =\bar{r}_{i}+\sum_{j=1, j \neq i}^{n} \bar{a}_{i j} e^{\alpha_{i j} x_{j}\left(t_{i}\right)}+\sum_{j=1, j \neq i}^{n} \bar{b}_{i j} e^{\beta_{i j} x_{j}\left(t_{i}-\tau_{i j}\right)} \\
& \quad+\sum_{j=1, j \neq i}^{n} \bar{c}_{i j}(t) e^{\gamma_{i j} x_{j}\left(t_{i}+s\right)} \\
& \leq \bar{r}_{i}+\sum_{j=1, j \neq i}^{n} \bar{a}_{i j} e^{\alpha_{j j} x_{j}\left(t_{j}\right)}+\sum_{j=1, j \neq i}^{n} \bar{b}_{i j} e^{\alpha_{j j} x_{j}\left(t_{j}\right)}
\end{aligned}
$$




$$
\begin{gathered}
+\sum_{j=1, j \neq i}^{n} \bar{c}_{i j}(t) e^{\alpha_{j j} x_{j}\left(t_{j}\right)} \\
=\bar{r}_{i}+\sum_{j=1, j \neq i}^{n}\left(\bar{a}_{i j}+\bar{b}_{i j}+\bar{c}_{i j}\right) e^{\alpha_{j j} x_{j}\left(t_{j}\right)} .
\end{gathered}
$$

Here we used $\left(H_{2}\right)$. Letting $\left(\underline{a}_{i i}+\underline{b}_{i i}\right) e^{\alpha_{i i} x_{i}\left(t_{i}\right)}=z_{i}\left(t_{i}\right)$, it follows from (20) that

$$
z_{i}\left(t_{i}\right) \leq \bar{r}_{i}+\sum_{j=1, j \neq i}^{n}\left(\bar{a}_{i j}+\bar{b}_{i j}+\bar{c}_{i j}\right) \underline{a}_{j j}^{-1} z_{j}\left(t_{j}\right),
$$

or

$$
z_{i}\left(t_{i}\right)-\sum_{j=1, j \neq i}^{n} \frac{\bar{a}_{i j}+\bar{b}_{i j}+\bar{c}_{i j}}{\underline{a}_{j j}} z_{j}\left(t_{j}\right) \leq \bar{r}_{i}
$$

which implies

$$
\begin{gathered}
\left(\begin{array}{cccc}
1 & -\frac{\bar{a}_{12}+\bar{b}_{12}+\bar{c}_{12}}{\underline{a}_{22}} & \cdots & -\frac{\bar{a}_{1 n}+\bar{b}_{1 n}+\bar{c}_{1 n}}{a_{n n}} \\
-\frac{\bar{a}_{21}+\bar{b}_{21}+\bar{c}_{21}}{\underline{a}_{11}} & 1 & \cdots & -\frac{\bar{a}_{2 n}+\bar{b}_{2 n}+\bar{c}_{2 n}}{\bar{a}_{n n}} \\
-\frac{\bar{a}_{n 1}+\bar{b}_{n 1}+\bar{c}_{n 1}}{\underline{a}_{11}} & -\frac{\bar{a}_{n 2}+\bar{b}_{n 2}+\bar{c}_{n 2}}{\underline{a}_{22}} & \cdots & 1
\end{array}\right) \\
\times\left(\begin{array}{c}
z_{1}\left(t_{1}\right) \\
z_{2}\left(t_{2}\right) \\
\cdots \\
z_{n}\left(t_{n}\right)
\end{array}\right) \leq\left(\begin{array}{c}
\bar{r}_{1} \\
\bar{r}_{2} \\
\cdots \\
\bar{r}_{n}
\end{array}\right)
\end{gathered}
$$

Set $D=\left(D_{1}, D_{2}, \ldots, D_{n}\right)^{T}=\left(\bar{r}_{1}, \bar{r}_{2}, \ldots, \bar{r}_{n}\right)^{T}$. It follows from (23) that

$$
(E-\mathscr{K})\left(z_{1}\left(t_{1}\right), z_{2}\left(t_{2}\right), \ldots, z_{n}\left(t_{n}\right)\right)^{T} \leq D .
$$

In view of $\rho(\mathscr{K})<1$ and Lemma $3,\left(E_{n}-\mathscr{K}\right)^{-1} \geq 0$. Let

$$
H=\left(\widetilde{h}_{1}, \widetilde{h}_{2}, \ldots, \widetilde{h}_{n}\right)^{T}:=(E-\mathscr{K})^{-1} D \geq 0 .
$$

Then it follows from (24) and (25) that

$$
\begin{aligned}
& \left(z_{1}\left(t_{1}\right), z_{2}\left(t_{2}\right), \ldots, z_{n}\left(t_{n}\right)\right)^{T} \leq H, \text { or } \\
& z_{i}\left(t_{i}\right) \leq \widetilde{h}_{i}, \quad i=1,2, \ldots, n,
\end{aligned}
$$

which implies

$$
\begin{array}{r}
\left|x_{i}(t)\right|_{0}=\max _{t \in[0, \omega]}\left|x_{i}(t)\right|=x_{i}\left(t_{i}\right) \leq \frac{1}{\alpha_{i i}} \ln \frac{\tilde{h}_{i}}{\underline{a}_{i i}+\underline{b}_{i i}}, \\
i=1,2, \ldots, n .
\end{array}
$$

On the other hand, it follows from (25) that

$$
\begin{aligned}
& (E-\mathscr{K}) H=D, \quad \text { or } \quad H=\mathscr{K} H+D, \text { that is, } \\
& \widetilde{h}_{i}=\sum_{j=1}^{n} \Gamma_{i j} \widetilde{h}_{j}+D_{i}, \quad i=1,2, \ldots, n .
\end{aligned}
$$

Estimating (16), by using (26) and (28), we have

$$
\begin{aligned}
& \left|\dot{x}_{i}(t)\right|_{0}=\lambda \mid r_{i}(t)-a_{i i}(t) e^{\alpha_{i i} x_{i}(t)}+\sum_{j=1, j \neq i}^{n} a_{i j}(t) e^{\alpha_{i j} x_{j}(t)} \\
& +\sum_{j=1, j \neq i}^{n} b_{i j}(t) e^{\beta_{i j} x_{j}\left(t-\tau_{i j}\right)} \\
& +\left.\sum_{j=1, j \neq i}^{n} c_{i j}(t) \int_{0}^{\infty} H_{i j}(s) e^{\gamma_{i j} x_{j}\left(t_{i}+s\right)} d s\right|_{0} \\
& \leq \bar{r}_{i}+\mid a_{i i}(t) e^{\alpha_{i i} x_{i}\left(t_{i}\right)}+\sum_{j=1, j \neq i}^{n} a_{i j}(t) e^{\alpha_{i j} x_{j}(t)} \\
& +\sum_{j=1, j \neq i}^{n} b_{i j}(t) e^{\beta_{i j} x_{j}\left(t_{j}\right)} \\
& +\left.\sum_{j=1, j \neq i}^{n} c_{i j}(t) \int_{0}^{\infty} H_{i j}(s) e^{\gamma_{i j} x_{j}\left(t_{i}+s\right)} d s\right|_{0} \\
& =\bar{r}_{i}+\sum_{j=1, j \neq i}^{n} \frac{\bar{a}_{i j}+\bar{b}_{i j}+\bar{c}_{i j}}{\underline{a}_{j j}} z_{j}\left(t_{j}\right)+\frac{\bar{a}_{i i}}{\underline{a}_{i i}} z_{i}\left(t_{i}\right) \\
& \leq D_{i}+\sum_{j=1}^{n} \Gamma_{i j} \widetilde{h}_{j}+\frac{\bar{a}_{i i}}{\underline{a}_{i i}} z_{i}\left(t_{i}\right) \leq \widetilde{h}_{i}+\frac{\bar{a}_{i i}}{\underline{a}_{i i}} \widetilde{h}_{i} \\
& =\left[1+\frac{\bar{a}_{i i}}{\underline{a}_{i i}}\right] \tilde{h}_{i} .
\end{aligned}
$$

We can choose a large enough real number $(d>1)$ such that

$$
\frac{1}{\alpha_{i i}} \ln \frac{d \tilde{h}_{i}}{\underline{a}_{i i}}>\frac{1}{\alpha_{i i}} \ln \frac{\tilde{h}_{i}}{\underline{a}_{i i}}+\left[1+\frac{\bar{a}_{i i}}{\underline{a}_{i i}}\right] \tilde{h}_{i} .
$$

Let $h_{i}=\left(1 / \alpha_{i i}\right) \ln \left(d \widetilde{h}_{i} / \underline{a}_{i i}\right)$. Then, for any solution of $L x=$ $\lambda N x$, we have $\left|x_{i}(t)\right|_{1}=\left|x_{i}(t)\right|_{0}+\left|\dot{x}_{i}(t)\right|_{0} \leq \ln \left(\widetilde{h}_{i} / \underline{a}_{i i}\right)+2 \widetilde{h}_{i}<$ $h_{i}$ for all $i=1,2, \ldots, n$. Obviously, $h_{i}$ are independent of $\lambda$ and the choice of $x(t)$. Thus, taking $h_{i}=\ln \left(d \widetilde{h}_{i} / \underline{a}_{i i}\right)$, the open subset $\Omega$ satisfies that $L x \neq \lambda N x$ for each $\lambda \in(0,1), x \in \partial \Omega \cap$ Dom $L$; that is, the open subset $\Omega$ satisfies the assumption (i) of Lemma 1.

Using similar arguments to Step 3 in [2], it is not difficult to show that, for each $x \in \partial \Omega \cap \operatorname{Ker} L, Q N x \neq 0$ and $\operatorname{deg}\{J Q N, \Omega \cap \operatorname{Ker} L, 0\} \neq 0$.

Hence, by Lemma 1, system (8) has at least one positive $\omega$-periodic solution in $\operatorname{Dom} L \cap \bar{\Omega}$. By (7), system (1) has at least one positive $\omega$-periodic solution, denoted by $\tilde{y}(t)$. This completes the proof of Theorem 4 .

\section{Conflict of Interests}

The authors declare that there is no conflict of interests regarding the publication of this paper. 


\section{References}

[1] M. Fan, K. Wang, and D. Jiang, "Existence and global attractivity of positive periodic solutions of periodic $n$-species LotkaVolterra competition systems with several deviating arguments," Mathematical Biosciences, vol. 160, no. 1, pp. 47-61, 1999.

[2] Y. H. Xia and M. Han, "New conditions on the existence and stability of periodic solution in lotka-volterra's population system," SIAM Journal on Applied Mathematics, vol. 69, no. 6, pp. 1580-1597, 2009.

[3] Y. Xia, "Periodic solution of certain nonlinear differential equations: via topological degree theory and matrix spectral theory," International Journal of Bifurcation and Chaos in Applied Sciences and Engineering, vol. 22, no. 8, Article ID 1250196, 17 pages, 2012.

[4] X. Gu, H. Wang, P. J. Y. Wong, and Y. Xia, "Existence and stability of periodic solution to delayed nonlinear differential equations," Abstract and Applied Analysis, vol. 2014, Article ID 156948, 12 pages, 2014.

[5] F. Yang, D. Jiang, and A. Ying, "Existence of positive solution of multidelays facultative mutualism system," Journal of Engineering Mathematics, vol. 3, pp. 64-68, 2002.

[6] F. Chen, J. Shi, and X. Chen, "Periodicity in a Lotka-Volterra facultative mutualism system with several delays," Journal of Engineering Mathematics, vol. 21, no. 3, pp. 403-409, 2004.

[7] Y. H. Xia, J. Cao, and S. S. Cheng, "Periodic solutions for a Lotka-Volterra mutualism system with several delays," Applied Mathematical Modelling, vol. 31, no. 9, pp. 1960-1969, 2007.

[8] R. E. Gaines and J. L. Mawhin, Coincidence Degree and Nonlinear Differential Equations, Springer, Berlin, Germany, 1977.final

[9] J. P. LaSalle, The Stability of Dynamical System, Society for Industrial and Applied Mathematics, Philadelphia, Pa, USA, 1976.

[10] A. Berman and R. J. Plemmons, Nonnegative Matrices in the Mathematical Science, Academic Press, New York, NY, USA, 1929. 


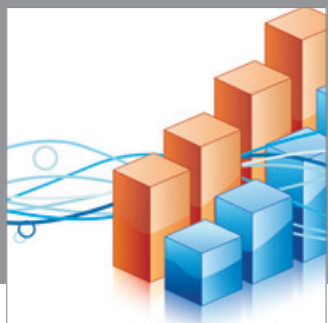

Advances in

Operations Research

mansans

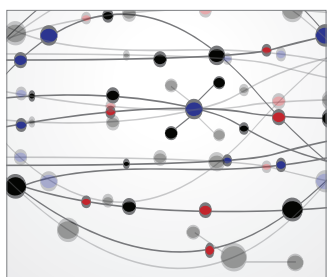

The Scientific World Journal
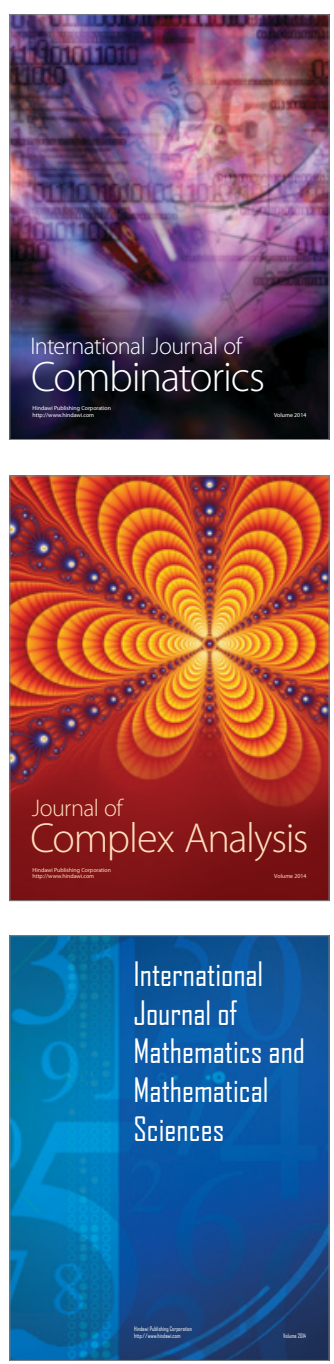
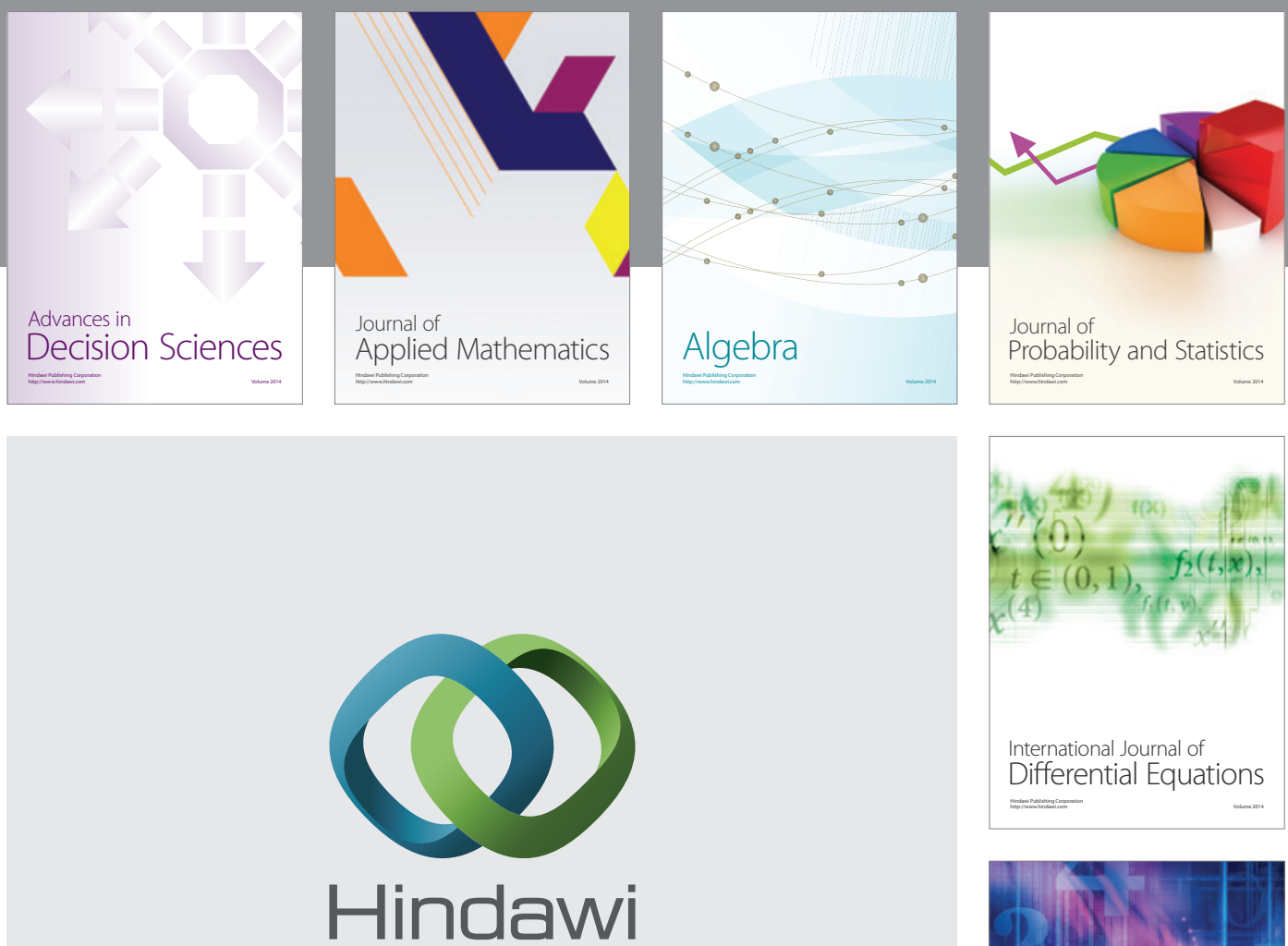

Submit your manuscripts at http://www.hindawi.com
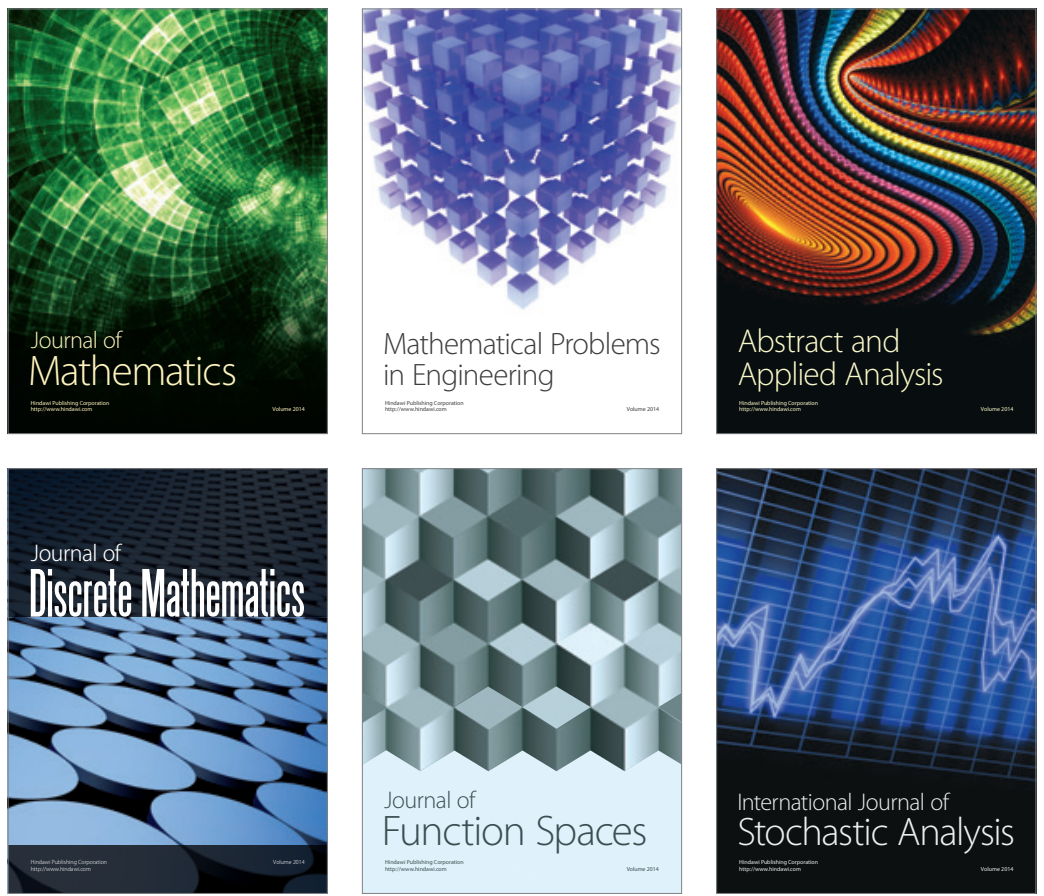

Journal of

Function Spaces

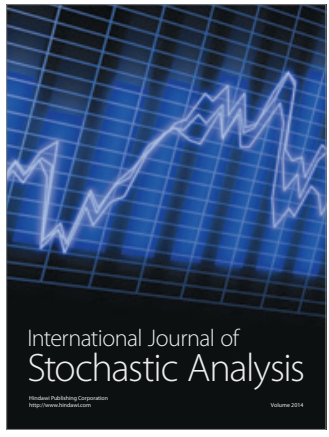

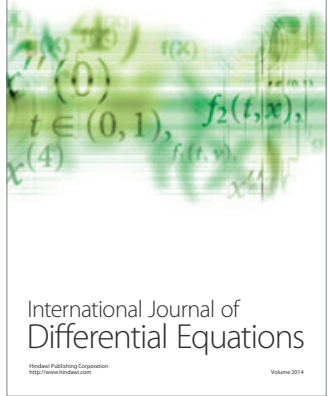
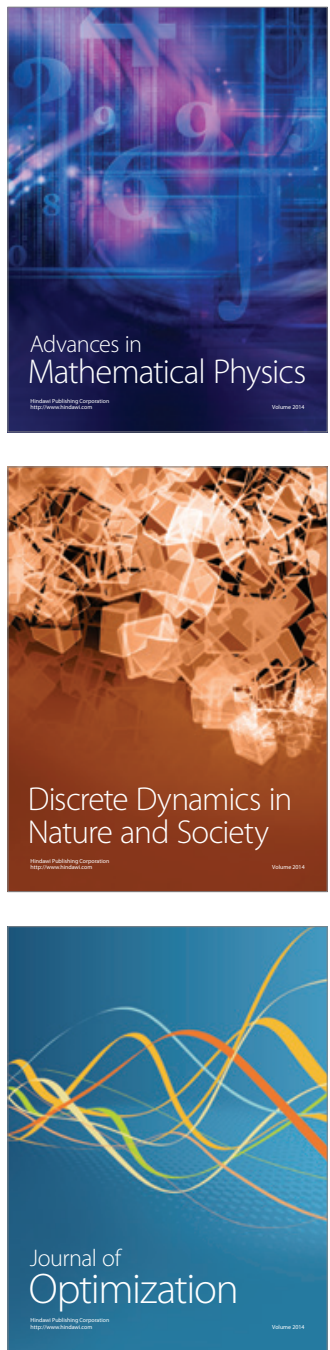